\title{
ABC of Blood Pressure Reduction
}

\section{THE SIZE OF THE PROBLEM}
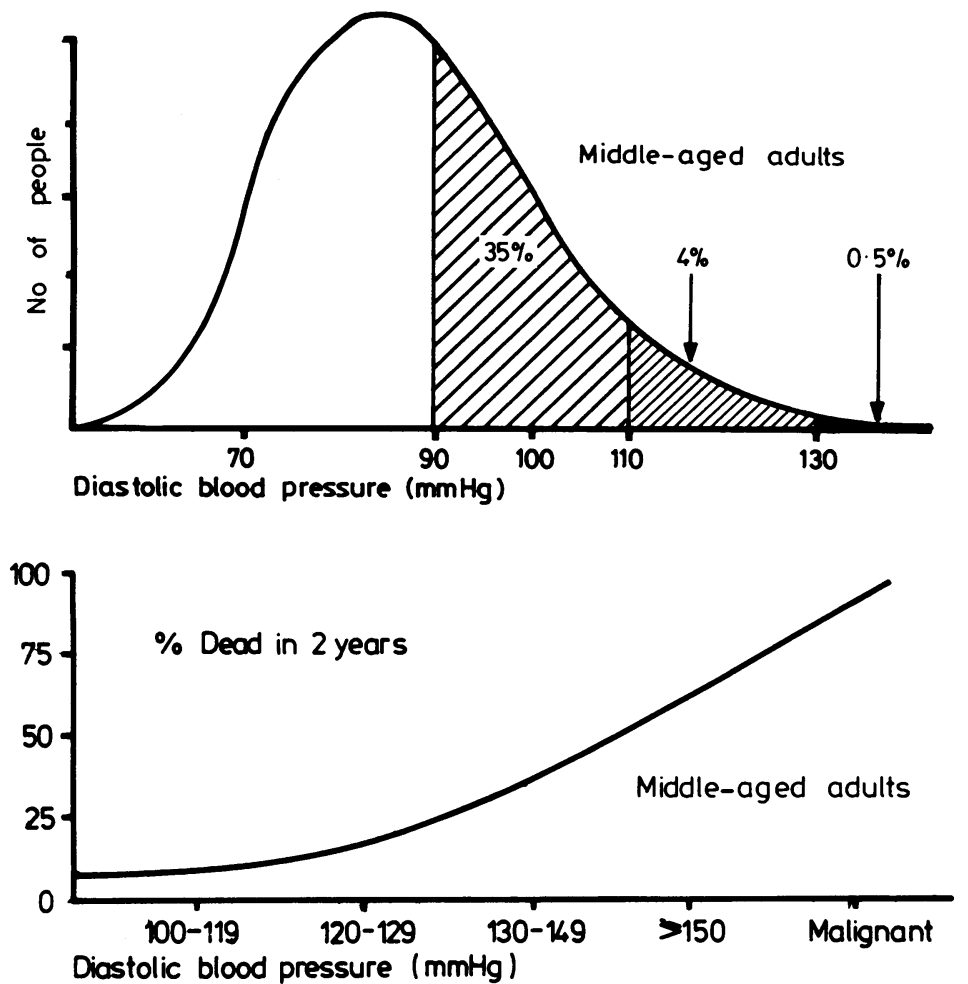

About $0.5 \%$ of the middle-aged population have diastolic blood pressures of $130 \mathrm{~mm} \mathrm{Hg}$ or more. If left untreated they have a prognosis worse than if they had cancer. A further $4 \%$ have diastolic pressures of 110 to $129 \mathrm{~mm} \mathrm{Hg}$, and about $20 \%$ of these will die in five years if left untreated. The gradient of risk extends to even lower pressures: people with diastolic pressures of $90 \mathrm{~mm} \mathrm{Hg}$ have a shorter life expectancy than those with pressures below this figure. A 35-year-old man with a diastolic pressure of $100 \mathrm{~mm} \mathrm{Hg}$ can expect a 16 -year reduction in life expectancy and so is likely to die before he retires.

Although the personal risk for patients with mild hypertension is much smaller than with the severe disease, most heart attacks and strokes occur in those with mild hypertension by virtue of the large numbers of cases. If treatment could abolish the consequences of mild hypertension mortality would fall dramatically and the population's life expectancy increase.

\section{Who should be treated?}

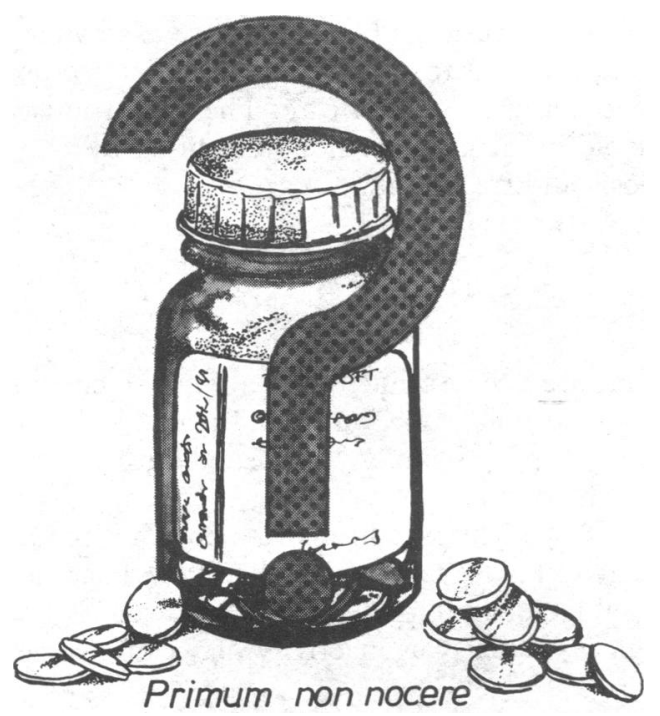

Because there is no clear-cut dividing line between hypertension and normotension, the most difficult clinical decision is to determine at which level of blood pressure drug treatment should be started. Evans and Rose's definition of hypertension as "that level of blood pressure above which investigation and treatment do more good than harm" aptly summarises the problem.

Remarkably, there have been only four randomised clinical trials of treatment. The first, published in 1964, concentrated on severe hypertension, and the most recent from Australia has looked at patients with diastolic pressures of 95-109 mm Hg. The British MRC trial of mild hypertension is still under way. 


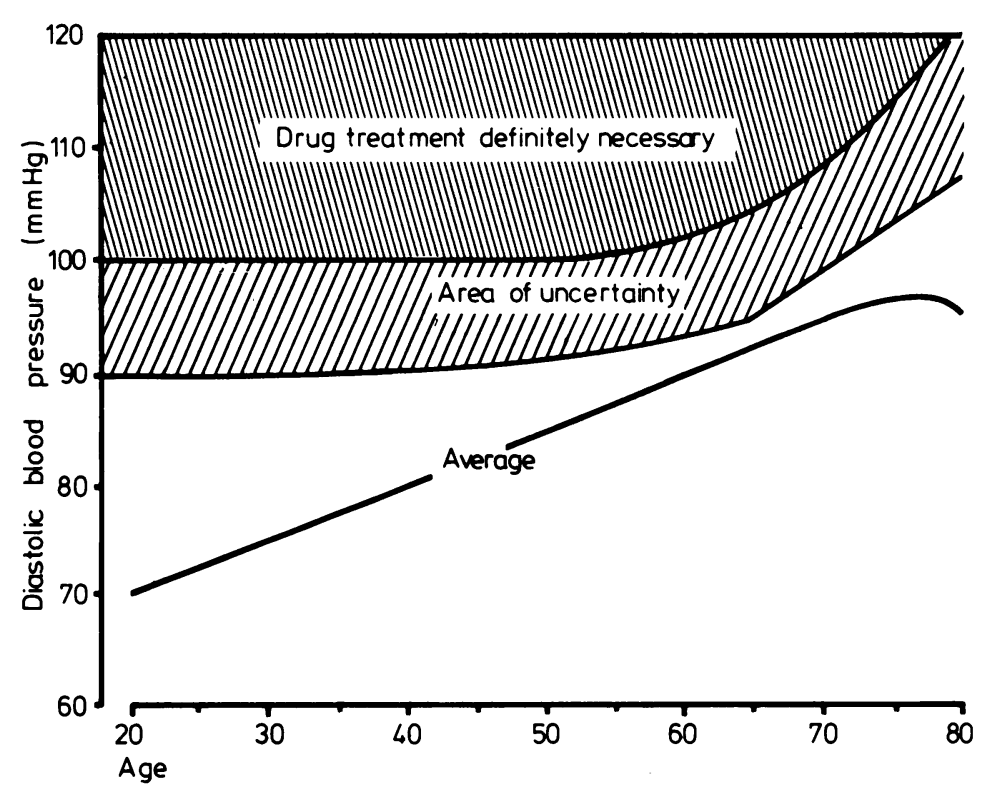

\section{Balancing risks and benefits}

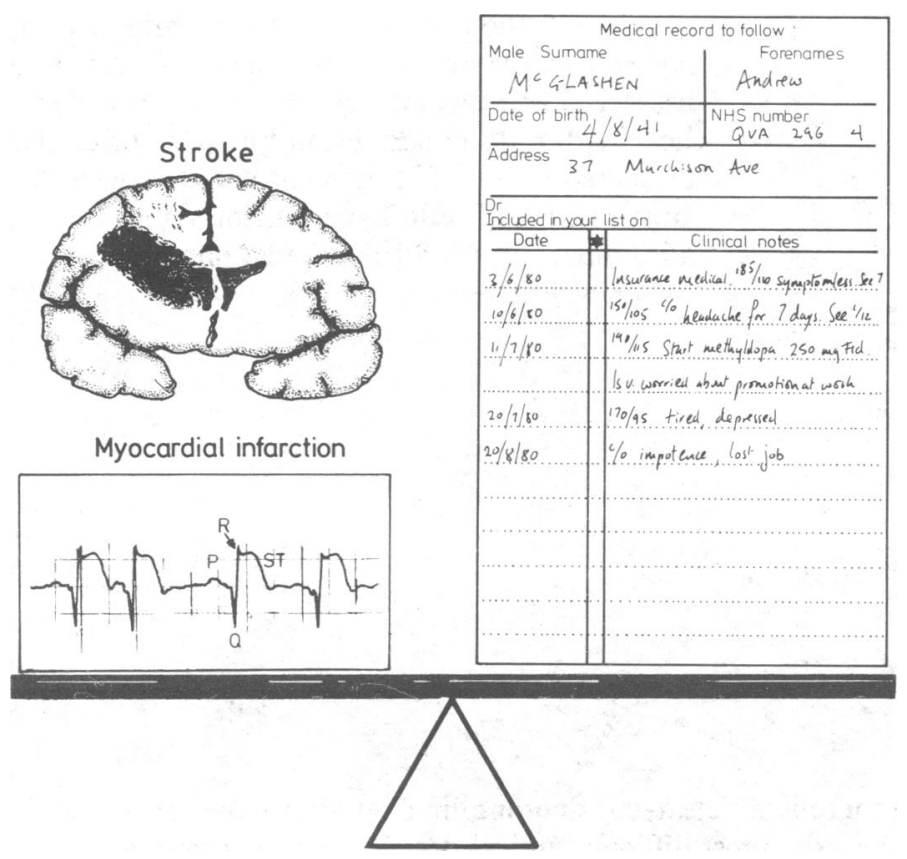

Taking published evidence into account, we suggest that in people aged under 65 treatment should definitely be started at diastolic pressures of consistently $100 \mathrm{~mm} \mathrm{Hg}$ or more. There is an area of uncertainty lying between $90 \mathrm{~mm} \mathrm{Hg}$ and 100 $\mathrm{mm} \mathrm{Hg}$, where treatment will depend on individual circumstances. Above the age of 65 the level of diastolic pressure at which treatment should be started rises. In 75-year-olds, for example, treatment need not be considered until the pressure is $105 \mathrm{~mm} \mathrm{Hg}$ and may not be necessary until it has reached $115 \mathrm{~mm} \mathrm{Hg}$.

The level of blood pressure where treatment should be started should be the average of several readings taken on different occasions, not just once. If there is any left ventricular enlargement, heart failure, or kidney damage or if there are other cardiovascular risk factors, such as cigarette smoking or hypercholesterolaemia, then the level of blood pressure at which treatment is necessary may be reduced by about $5 \mathrm{~mm} \mathrm{Hg}$. In patients under 65 years treatment should aim at keeping the pressure below $140 / 90 \mathrm{~mm} \mathrm{Hg}$. Reduction to below this level is not harmful as long as precipitate drops in pressure are avoided.

Having decided to treat the patient, the doctor must tell the patient the reasoning behind all the trouble and extra stress to which he will be subjected. A balance has to be struck in the patient's mind between the benefits of treatment and its inconveniences. The benefits are the hoped for prolongation of life, with freedom from stroke, heart failure, and renal complications and, possibly, a lowered risk of heart attack. These must be weighed against the repeated visits to the surgery, numerous blood pressure readings, initial blood tests, and other investigations; the chore of taking tablets every day; and the side effects of the tablets. It has to be emphasised that any symptoms the patient has are probably unrelated to his high blood pressure and that treatment, far from relieving his symptoms, may increase them because of drug side effects. The patient's co-operation in the management of his blood pressure is so vital that time spent at this stage reaps ample rewards in the succeeding decades of care. The doctor must also avoid inducing hypochondria caused by diagnosis and treatment.

\section{The first step in treatment}

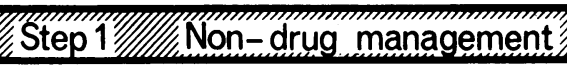

Once secondary hypertension has been excluded and a baseline chest radiograph, electrocardiograph, and renal function value obtained, the other risk factors, which together with hypertension are associated with ischaemic heart disease, should be tackled.

Trying to influence a patient's lifestyle to reduce stress does not reduce blood pressure in the long term and is fraught with difficulties. There is no evidence that anxiety is a causal factor in hypertension. Hard work is not harmful and may be beneficial, particularly as inactivity may cause some gain in weight. 


\section{Obesity}

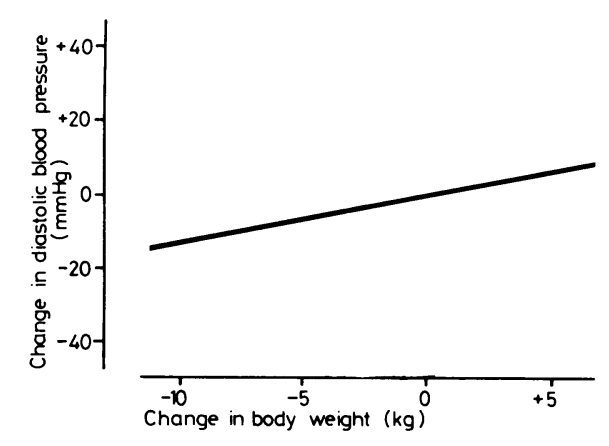

\section{Salt}

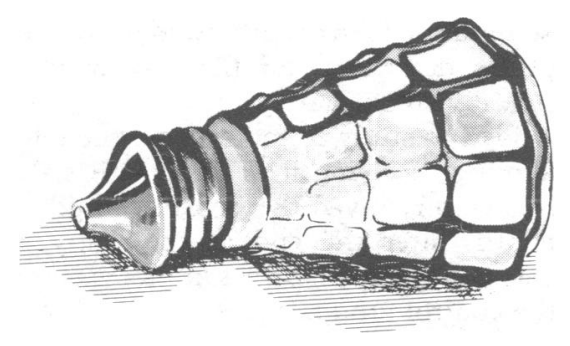

\section{Alcohol}

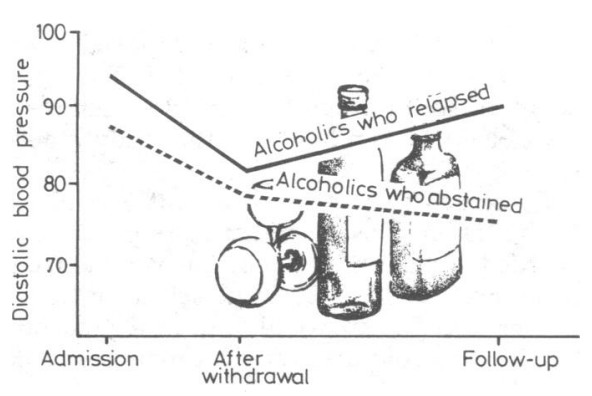

\section{Cigarette smoking}

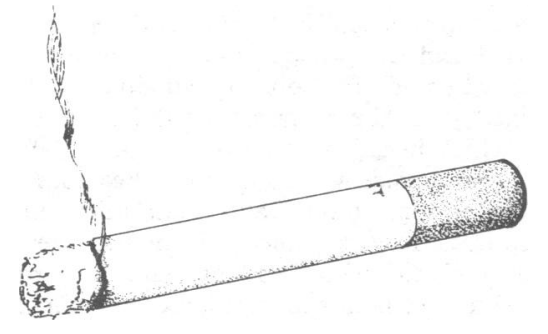

Obesity is strongly associated with hypertension, and a reduction in weight produces a fall in blood pressure. Every attempt should be made to get the obese patient to diet so that his weight falls within the norm for his height and build.
Severe reduction in salt intake lowers high blood pressure but also renders the diet unpalatable. A less drastic reduction in salt intake may cause a modest fall in blood pressure and a reduction of the dietary salt to less than $100 \mathrm{mmol} /$ day may be recommended. This is best achieved by asking the patient to stop adding salt to his food after it has been cooked.

Though the link between salt and pressure is unproved, there is more evidence in its favour than against, and moderate salt restriction does not harm.
Because of the strong association of substantial intakes of alcohol with hypertension and the fact that abstinence leads to a fall in blood pressure, all hypertensive patients should curtail their alcohol intake to no more than two drinks a day. A moderate alcohol intake may protect against ischaemic heart disease. The association between alcohol and hypertension, though well documented, is not commonly appreciated and it cannot therefore be overemphasised.
Though studies have failed to show a link between benign hypertension and cigarette smoking, a strong association does exist with malignant hypertension. For this reason, and because cigarette smoking has an independent role in all forms of vascular disease, hypertensive patients should be persuaded to stop smoking completely. This may lead to a small increase in weight and occasionally to a rise in blood pressure. Despite this the priority is to stop smoking.

\section{Drugs inducing hypertension}

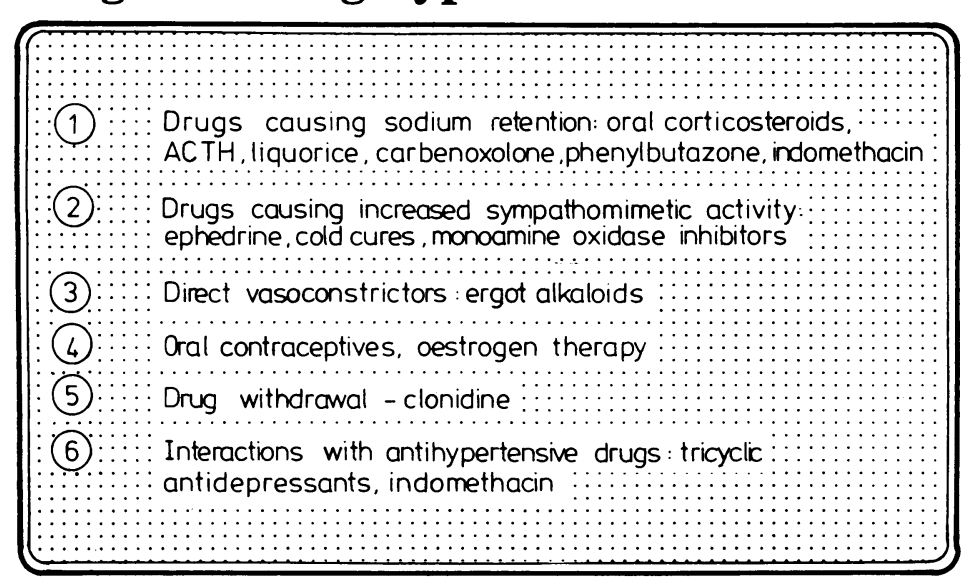

Excluding drugs that cause or aggravate hypertension is an important part of the initial assessment of the hypertensive patient. Sometimes it is not possible to discontinue these other drugs, but when possible alternative forms of treatment should be used.

Dr Liam Bannan, BSC, MRCPI, is an honorary research fellow, University of Birmngham, Dr D G Beevers, MD, MRCP, a senior lecturer in medicine, University of Birmingham, and Dr Noel Wright, MB, FRCPED, a consultant physician, Dudley Road Hospital, Birmingham. 